\title{
Perspective
}

PERSPECTIVE Actualité en histoire de l'art

$1 \mid 2020$

Japon

\section{Rosetsu, ses Cinq cents Arhat, et la culture des expositions dans le Kyōto du XVIII ${ }^{\mathrm{e}}$ siècle}

Rosetsu's One-Inch Square Picture and the Culture of Exhibitions in $18^{\text {th }}$-century Kyōto

Rosetsu, seine Fünfhundert Arhat und die Ausstellungskultur im Kyōto des

18. Jahrhunderts

Rosetsu, i suoi Cinquecento Arhat e la cultura delle esposizioni nella Kyōto del XVIII secolo

Rosetsu, sus Quinientos Arhat, y la cultura de las exposiciones en el Kyōto del siglo XVIII

\section{Matthew McKelway}

Traducteur : Étienne Gomez

\section{OpenEdition}

\section{Journals}

Édition électronique

URL : http://journals.openedition.org/perspective/18586

DOI : 10.4000/perspective. 18586

ISSN : 2269-7721

Éditeur

Institut national d'histoire de l'art

Édition imprimée

Date de publication : 5 juin 2020

Pagination : 201-220

ISBN : 978-2-917902-89-9

ISSN : $1777-7852$

Référence électronique

Matthew McKelway, «Rosetsu, ses Cinq cents Arhat, et la culture des expositions dans le Kyōto du xvIII ${ }^{e}$ siècle », Perspective [En ligne], 1 | 2020, mis en ligne le 30 décembre 2020, consulté le 25 janvier 2021. URL : http://journals.openedition.org/perspective/18586 ; DOI : https://doi.org/10.4000/ perspective. 18586 


\title{
Rosetsu, ses Cinq cents Arhat, et la culture des expositions dans le Kyōto du XVIII ${ }^{\mathrm{e}}$ siècle
}

\author{
Matthew McKelway
}

Le 10 septembre 1799, Minagawa Kien (1734-1807), l'éminent savant confucéen de Kyōto de la fin du XVIII ${ }^{\mathrm{e}}$ siècle (on lui doit plus d'une dizaine de livres), décrivit brièvement une peinture remarquable de son proche ami, le peintre Nagasawa Rosetsu (1754-1799) :

Nagasawa Rosetsu était déjà un peintre réputé pour son talent, et à chacune
de ses expositions, ses œuvres gagnaient en merveille. L'année dernière, à l'occasion
de l'exposition estivale du quatrième mois lunaire, il a représenté cinq cents arhat
sur un carré de trois centimètres sur trois, chacun en pied, avec aussi des silhouettes
de lion, d'éléphant, de dragon, et de tigre. Malgré l'extrême petitesse et minutie
de la représentation, chaque élément apparaissait avec sa forme propre, et son pinceau
manifestait un raffinement accompli. C'était une véritable merveille pour les yeux ${ }^{1}$.

Ces quelques lignes représentent un cas relativement rare de commentaire esthétique, par un contemporain, d'une peinture japonaise antérieure à l'époque moderne et parvenue jusqu'à nous. La nouvelle de la réapparition de cette peinture a fait sensation au Japon en 2010, car on ne l'avait pas revue depuis 1928 et on ne la connaissait que par une photographie en noir et blanc publiée dans un catalogue de vente à cette date. La peinture Cinq cents Arhat (方寸五百羅漢), qui suscite un vif intérêt depuis sa première exposition moderne en 2011, a été présentée à l'automne 2018 dans l'exposition Rosetsu - D'un pinceau impétueux que j'ai codirigée à Zurich², et au printemps 2019 dans l'exposition Les Lignées d'excentriques - Le monde miraculeux de la peinture Edo à Tōkyō ${ }^{3}$. Comme il arrive souvent au Japon, on ne sait presque rien de sa provenance ni de ses propriétaires jusqu'au moment de sa réapparition. Fasciné par l'idée aussi bien que par l'exécution de cette peinture âgée de deux cent vingt ans, l'artiste Murakami Takashi, du mouvement Superflat, en a récemment fait l'acquisition, puis une copie, réalisant par ailleurs une peinture murale sur le même thème ${ }^{4}$.

J'aimerais faire de Cinq cents Arhat le point de départ d'une enquête en deux temps sur l'art de Nagasawa Rosetsu et sur ce que j'appellerai une " culture des expositions " naissante dans le Japon de la fin de la période d'Edo, qui a stimulé sa créativité. Depuis un demi-siècle, Rosetsu est considéré comme un représentant proto-moderne dans la "lignée d'excentriques » de la période d'Edo (vers 1600 - 1868), mais j'aimerais 


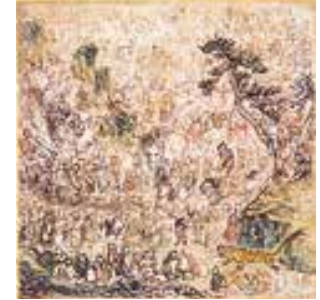

1a-b. Rosetsu Nagasawa, Cinq cents Arhat, taille réelle [a], et agrandissement [b], encre et couleur sur papier, 3,03 × 3,03 cm, 1798, collection privée.

montrer qu'au-delà d'un environnement particulièrement créatif, c'est le contexte sociohistorique des expositions publiques d'œuvres de peintres (et de calligraphes) contemporains qui l'a poussé à réaliser cette œuvre insolite. Inscrivant mon étude dans la perspective de recherches récentes sur les réseaux des peintres et sur leurs communautés de mécènes ${ }^{5}$, il me semble possible de soutenir que l'évolution de Rosetsu et de ses contemporains vers des œuvres toujours plus saisissantes sur le plan visuel, en particulier pendant l'ère Kansei (1789-1801), est intrinsèquement liée à l'essor de nouvelles pratiques d'exposition - ce terme désignant les divers milieux où l'on montrait des images. J'espère que l'article qui suit, qui part de cette minuscule peinture de Rosetsu qu'est Cinq cents Arhat, contribuera à un réexamen plus général de la créativité des peintres dans le Kyōto prémoderne.

Depuis la publication, il y a une cinquantaine d'années, de l'ouvrage de Tsuji Nobuo intitulé Kisō no keifu [Lignées d'excentriques], la notion d' « excentricité " occupe une grande place dans l'histoire de l'art japonais du début de l'époque moderne. La catégorie des « excentriques " n'avait rien de neuf, puisque dès 1790, Ban Kōkei publiait sous le titre Kinsei kijin den [Vies des excentriques de l'époque moderne] un recueil de plus d'une centaine de notices biographiques sur des personnalités remarquables, dont des hommes de lettres aussi bien que des paysans et d'autres roturiers ${ }^{6}$. Dans ses travaux, entrepris en 1968 sous la forme d'une série de courts essais pour la revue d'art contemporain Bijutsu techō, le professeur Tsuji se revendiquait pourtant moins du recueil d'anecdotes de Ban Kōkei que de récentes études sur la peinture chinoise du début de l'époque moderne ${ }^{7}$. On a d'ailleurs fait remarquer qu'aucun des sujets d'étude de Tsuji, de Kano Sansetsu à Iwasa Matabei ou d'Itō Jakuchū à Rosetsu, ne figurait dans le livre de Ban Kōkei. C'est ce choix pertinent qui a permis à Tsuji de jeter sur cet ensemble de peintres un regard à la fois nouveau et conforme à une manière plus ancienne de les voir, puisqu'elle remontait au XVIII ${ }^{\mathrm{e}}$ siècle. L'impact durable de la classification des " excentriques » de Tsuji, désormais canonique, tient au fait qu'elle a introduit la possibilité d'une analyse de l'art japonais indépendante des normes de la méthodologie historique en usage pour l'art occidental. Elle a cependant les mêmes limitations que n'importe quel canon, en ce qu'elle laisse beaucoup de côté.

Mon objectif ici n'est pourtant pas de signaler le cas de peintres de mérite qui auraient jusque-là échappé à notre attention (et à celle de Tsuji) : les musées et les chercheurs en art japonais le font déjà très bien. Il ne s'agira pas non plus de faire une incursion dans le domaine de l'historiographie de l'art japonais. Mon souhait est plutôt de situer les œuvres de l'un de ces artistes, Rosetsu, dans le contexte original des expositions et des présentations publiques, car je crois que c'est dans ce contexte de compétition et de collaboration que des dizaines, voire des centaines de maîtres du pinceau établis dans ces centres urbains d'une grande densité qu'étaient Kyōto, Edo et Ōsaka, ont trouvé leur style pictural. Je rejoins l'avis de certains qui ont soutenu que le XVIII ${ }^{e}$ siècle avait été à Kyōto une période d'effervescence, de créativité et de diversité toute particulière pour la peinture, sans équivalent au Japon ni dans de nombreux pays, avant ou après, et j'espère ici envisager Rosetsu dans ce contexte.

\section{Les arhat et la culture des expositions}

La peinture de Rosetsu est un tour de force matériel et conceptuel. Dans un carré de 3 centimètres sur 3, il a peint des dizaines et des dizaines d'arhat (fig. la-b). Je ne les ai pas comptés, mais on voit assez bien se dégager une composition complexe 


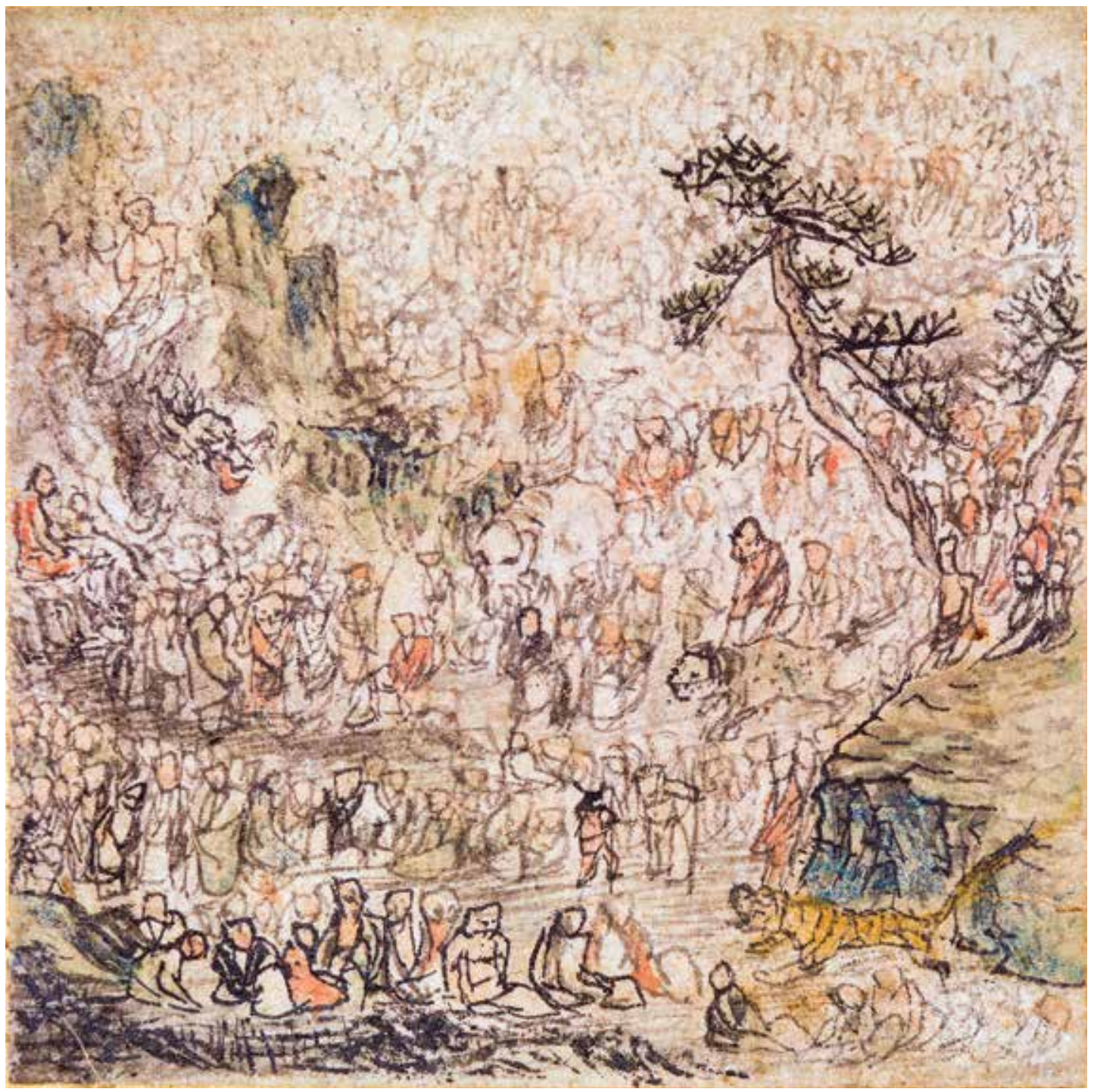

de silhouettes simplifiées, la plupart dépourvues de caractéristiques faciales, vêtues de robes amples et réparties en petits groupes sur la surface entière de l'œuvre. Les arhat du premier plan sont les plus précis en même temps que ceux dont le trait est le plus appuyé ; suivant les lois de la perspective aérienne, les plus éloignés apparaissent flous. Un promontoire rocheux, bleu et vert pâle, se dresse sur le devant à droite, surmonté de deux pins étendant leurs branches. Des escarpements plus prononcés se dressent dans le coin supérieur gauche. Un sentier, évoqué par une succession de traits horizontaux de couleur gris sombre, oblique du bord en bas à droite vers le milieu de la peinture, jusqu'au bord gauche. Pour compléter la scène, quatre créatures sont représentées : un tigre, un lion, un éléphant, et un dragon.

Les arhat étaient depuis longtemps un sujet d'inspiration majeur à l'époque de Rosetsu. Depuis le succès qu'ils avaient connu en Chine plusieurs siècles auparavant, ces êtres accomplis, réputés pour avoir été des disciples de Bouddha dotés d'une exceptionnelle prajña, la sagesse transcendante, ils étaient abondamment représentés par la peinture 
et la sculpture ${ }^{8}$. Dans la peinture, leur sagesse apparaissait souvent à travers la mise en scène de bonnes actions, comme les aumônes, ou de pouvoirs surnaturels, comme la transfiguration ou l'apparition sous la forme de Bodhisattvas. Selon une tradition, les arhat demeuraient ici-bas pour protéger la Vraie Loi du Bouddha Sakyamuni après sa mort et son entrée dans le nirvana. Dans le bouddhisme Mahāyāna (du « Grand Véhicule ») répandu en Asie de l'Est, les arhat sont vénérés en tant que modèles de la voie vers l'éveil. Parmi les plus anciennes peintures d'arhat connues, on peut mentionner une série de seize rouleaux attribués (mais ce sont probablement des copies) au moine-peintre Guanxiu (832-912), de la fin de la dynastie Tang, dont certains font partie de la collection impériale japonaise ${ }^{9}$ (fig. 2). Selon un texte du début de la dynastie Song, Yizhou minghualu [Vies de peintres éminents du Sichuan], Guanxiu a vu en rêve seize arhat absurdement déformés, dont il a peint à son réveil les étranges semblances ${ }^{10}$. Les représentations d'arhat suivent souvent ce célèbre précédent et leur prête des traits exagérés ou grotesques. Les noms des arhat ont été recensés dans un texte traduit par Xuanzang, Nandimitrāvadāna

2. Guanxiu (attr.), Arhat, dynastie Yuan, XIV siècle, rouleau suspendu, encre sur soie, 110,2 × 50,9 cm, Tōkyō, musée Nezu.

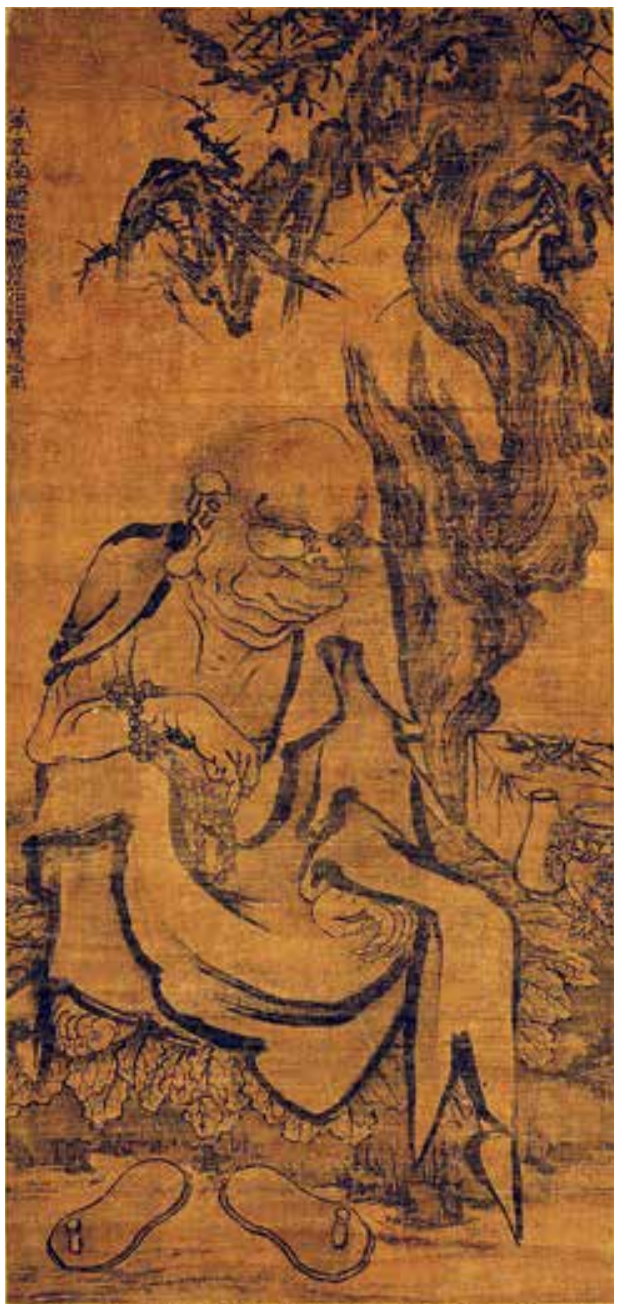

[Traité sur la résidence de la Loi, exposé par le grand arhat Nadimitra], quatre d'entre eux étant représentés dans la peinture de Rosetsu : l'arhat à dos d'éléphant, l'arhat dompteur de tigre, l'arhat dompteur de dragon, et l'arhat au lion riant.

Selon des combinaisons variées, par groupes de seize, de dix-huit, voire de cinq cents, les arhat occupent une place éminente dans les temples zen du Japon, le plus célèbre étant le monastère zen de Daitokuji où est aujourd'hui exposée, tous les ans, une partie des cent rouleaux suspendus des peintres chinois Lin Tinggui (actif 1174-1189) et Zhou Jichang (fin du XII ${ }^{\mathrm{e}}$ siècle) connus sous le nom de Cinq cents Luohan (l'autre est répartie entre deux musées américains). Il se peut que Rosetsu les ait vus, et qu'il ait vu d'autres œuvres de son époque, comme les peintures sur panneau coulissant et les sculptures sur pierre qu'Ikeno Taiga (1723-1776) et Itō Jakuchū (1716-1800) ont respectivement réalisées pour les monastères de Manpukuji vers 1772 et de Sekihōji à la fin des années 1780. Si le choix de support et de technique de Taiga, qui a procédé exclusivement à l'aide de ses doigts et de ses ongles sur une surface démesurée (fig. 3), semble en harmonie avec la nature céleste des arhat, l'abstraction de Jakuchū, qui a délaissé le pinceau pour la pierre brute, fait ressortir leur étrangeté physique.

Dans la description qu'il donne en 1799 des Cinq cents Arhat de Rosetsu, Kien dit qu' « à chacune de ses expositions, ses œuvres gagnaient en merveille ", et que " l'année dernière, à l'occasion de l'exposition estivale du quatrième mois lunaire, il a représenté cinq cents arhat sur un carré de trois centimètres sur trois ". Il attire donc notre attention sur le fait que Rosetsu présentait régulièrement son travail dans des 

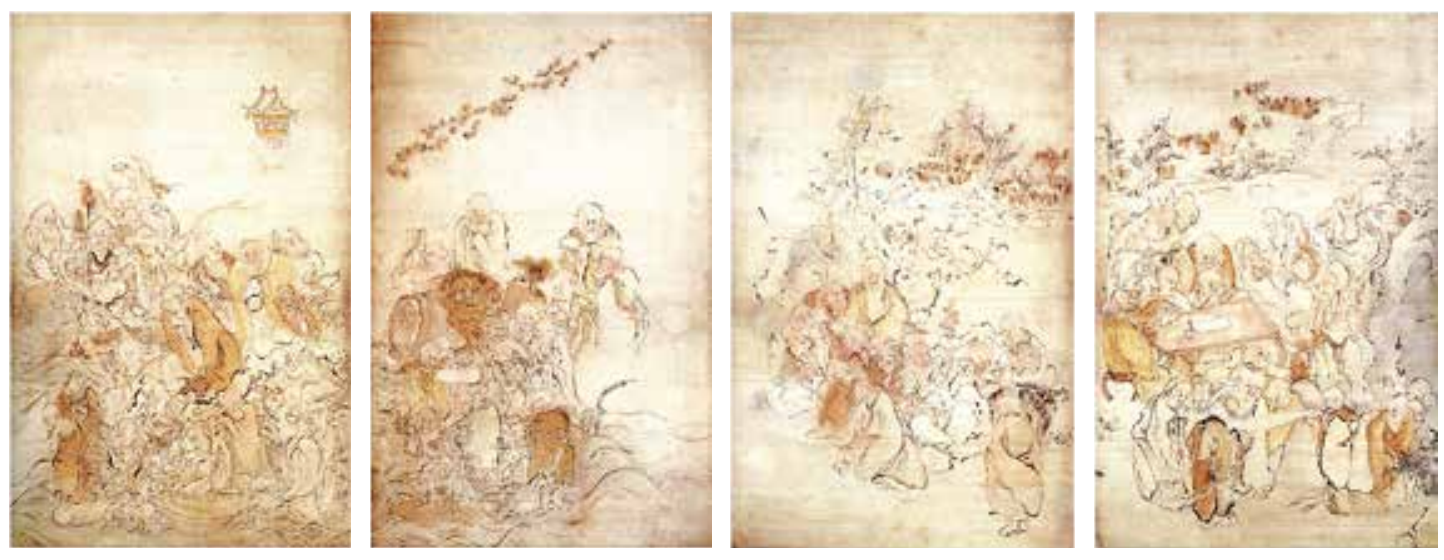

expositions, et sur le lien entre la fréquence de telles manifestations et le degré de "merveille " (le mot ki signifie par ailleurs " étrangeté " ou " excentricité ") 3. Ikeno Taiga, Cinq cents Arhat, 1765, quatre panneaux, $180 \times 115 \mathrm{~cm}$ chacun, Kyōto, Tō-hōjō, temple Manpuku-ji.

des œuvres présentées. Exécuté l'année ayant précédé la mort de Rosetsu, le rouleau des Cinq cents Arhat apparaît ainsi, à en juger par la remarque de Kien, comme un point culminant dans son aspiration à réaliser des œuvres capables de surprendre et de réjouir leurs spectateurs. Quel est donc le sens de cette œuvre?

Dans le même texte, Kien évoque brièvement l'origine des expositions semestrielles organisées dans les hauteurs orientales de Kyōto :

Depuis longtemps, dans l'espoir de promouvoir la peinture et la calligraphie à Kyōto, Ōsaka et Edo, j'invite chaque année des artistes que je connais à se réunir à Higashiyama à une certaine date au printemps et à l'automne, et à apporter leurs œuvres. J'appelle ces événements les Shinshoga tenkan [Expositions de calligraphies et de peintures nouvelles].

De Kansei 4 (1792) à l'année passée, Kansei 10 (1798), il y en a eu quatorze, et chaque fois ce sont entre trois et quatre cents rouleaux qui y ont été exposés. Leur renommée a attiré des étrangers, ce qui en a fait un véritable succès. Les calligraphes et peintres de Kyōto qui venaient en masse à ces événements rivalisaient pour créer des œuvres originales et bizarres ${ }^{11}$.

D'après ce passage, les Expositions de calligraphies et de peintures nouvelles ont commencé en 1792 et se sont poursuivies pendant sept ans, au printemps et à l'automne, soit quatorze fois en tout. Kien situe ces événements dans les " montagnes de l'est " (higashi-yama) de Kyōto. Il faut remarquer que si d'autres expositions, comme l'hommage à Ashikaga Yoshimasa dans le temple Ginkakuji en 1789, s'étaient déjà tenues auparavant, l'aspect systématique, deux fois par an, au même endroit, avec les mêmes mécènes, de celles-ci était novateur ${ }^{12}$. Les expositions organisées pour commémorer les carrières d'éminents artistes et intellectuels, devenues courantes à Kyōto, s'étendaient alors à Ōsaka et à Edo au début des années 1800, avec des hommages à Ikeno Taiga en 1800, Maruyama Ōkyo en 1807, Nagasawa Rosetsu lui-même en 1810, Kimura Kenkadō en 1813, ou encore Ogata Kōrin en 1815.

Seuls deux catalogues nous sont parvenus des quatorze expositions d'Higashiyama évoquées par Kien (1796 et 1797), auxquels viennent encore s'ajouter deux catalogues qui correspondent à des expositions du début du XIX siècle, dont l'une est ultérieure à sa mort (1806 et 1825) ${ }^{13}$. On ne sait pas si les publications sont apparues dès les premières 


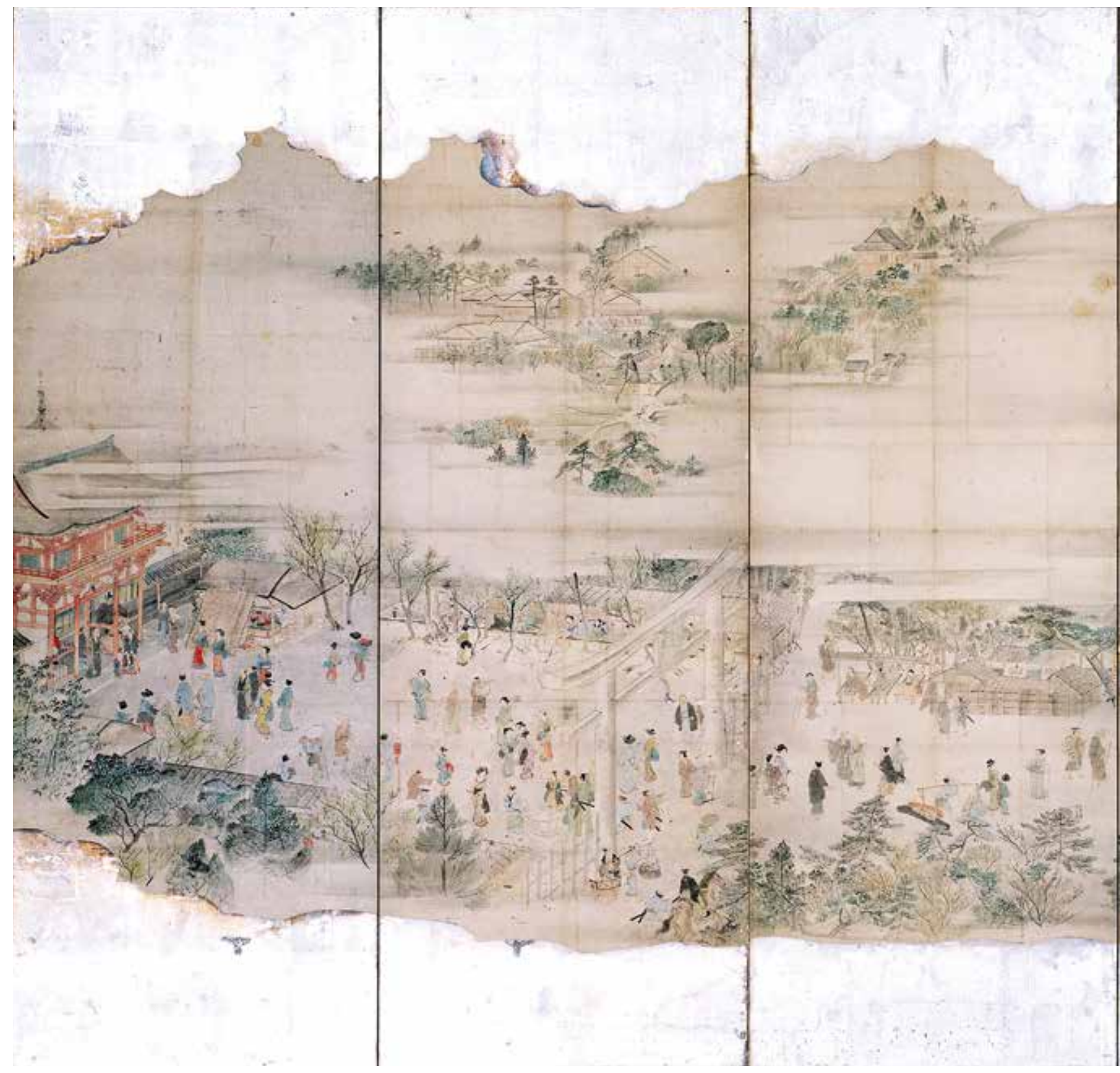




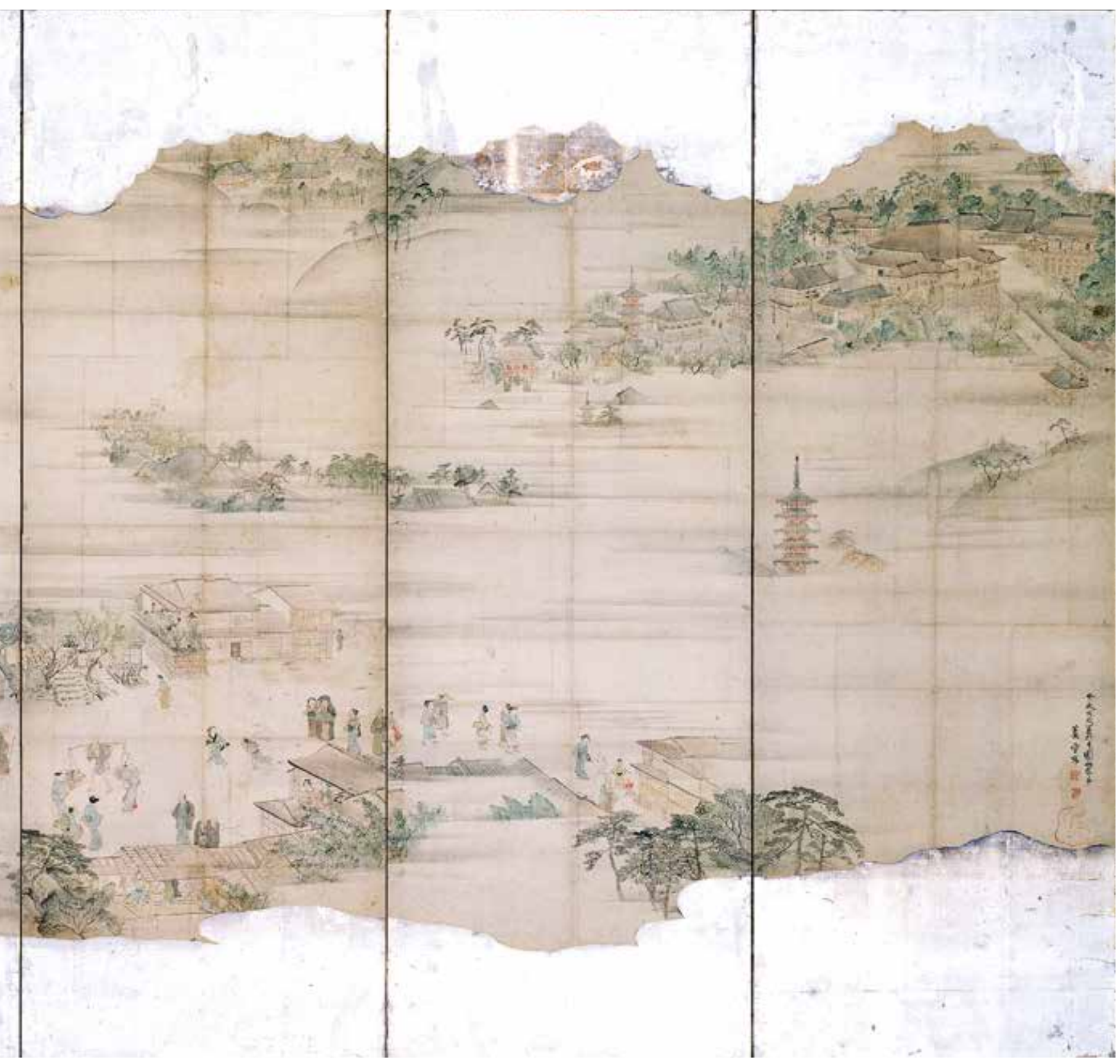


4. Rosetsu Nagasawa, Vues de Higashiyama, fin des années 1770, paravent de six panneaux, encre et couleur sur papier, $165,5 \times 344 \mathrm{~cm}$ collection privée.

Les pavillons Tazō'an et Tanryō sont représentés dans la partie supérieure du deuxième panneau (à partir de la gauche). expositions ou seulement par la suite, mais la liste de 212 œuvres de calligraphie et de peinture présentée dans le catalogue de 1796, avec les titres des œuvres et les noms des artistes, suggère un format bien établi dans l'inventaire des œuvres exposées. Malgré la rareté de ces catalogues, d'autres sources, au premier rang desquelles les écrits de Kien, donnent des informations précises sur les lieux et dates de plusieurs de ces expositions ${ }^{14}$. Quatre lieux différents sont mentionnés, les temples Kiyomizudera et Sōrinji, et les pavillons Tazō'an et Tanryō. Mis à part le temple Kiyomizudera, situé à une vingtaine de minutes à pied vers le sud, ces lieux se trouvaient dans le voisinage immédiat, le long du parc Maruyama actuel. Les représentations contemporaines de ces événements nous donnent une image de ces lieux, vus de plus ou moins loin. Un paravent peint par Rosetsu représente ainsi un ensemble de bâtiments perchés sur les hauteurs de Higashiyama, au milieu de collines et de bosquets arborés (fig. 4). On reconnaît aisément Kiyomizudera à l'extrême droite. Sōrinji (ou Chōrakuji), identifiable à son toit de tuiles, apparaît sur le quatrième panneau, à droite, sur une autre colline, tandis que les bâtiments situés à gauche sur le cinquième panneau forment le temple An'yōji. C'est là que se trouvaient les pavillons Tazō'an et Tanryō (aussi appelés Karaku'an), qui, comme les quatre autres sous-temples d'An'yōji, servaient à l'origine à loger les pèlerins et furent transformés en hôtels et en restaurants à la suite de la Restauration de Meiji. Ils ont déjà les caractéristiques de lieux de plaisance au XVIII ${ }^{\mathrm{e}}$ siècle. Rosetsu en fait un groupe de constructions légères surmontées de toits en bardeaux, plus proches de ryōtei modernes que de salles de dévotion. Un guide de Kyōto publié en 1780, intitulé Miyako meisho zue, vante leurs "feuilles vertes, pelouses parfumées, et fleurs perpétuelles", tandis qu'un guide complémentaire et détaillé des jardins panoramiques de Kyōto publié en 1799, Miyako rinsen meishō zue, consacre plusieurs pages illustrées à la description de ces auberges, à commencer par le " Tan-no-ryō " qui, avec ses divers niveaux, ses salles très spacieuses et ses vues sur le jardin, semble parfaitement adapté aux expositions de peintures et de calligraphies évoquées par Kien (fig. 5).

Tim Clark, dans son étude récente sur l'exposition tenue en hommage à Itō Jakuchū de 1885, complète la vision que l'on peut avoir de ces expositions grâce à plusieurs illustrations tirées d'un album de Kawamura Bunpō (1779-1821), un jeune contemporain de Rosetsu et de Kien invité à Higashiyama ${ }^{15}$. Tout en renvoyant à cette étude pour davantage de détail, je me contenterai ici de faire allusion à la série intitulée "Shoga tenkan zu " [Images d'une exposition de peintures et de calligraphies], où Bunpō semble transposer quelque chose comme la relation de son témoignage oculaire, à partir de ses souvenirs personnels de ces événements, en parant de costumes chinois ses personnages qui emballent, déroulent, accrochent, et estiment les œuvres réunies dans un univers raffiné et exotique (fig. 6a-b). La vision grossissante que nous donnent tour à tour le paravent de Rosetsu, les illustrations du guide, et les images de Bunpō nous aident ainsi à donner corps aux témoignages de Kien.

Il y a par ailleurs de nombreuses leçons à tirer des catalogues qui nous sont parvenus. Le catalogue de 1796, qui dresse un inventaire de 212 œuvres de peinture et de calligraphie, évoque ainsi un certain Shōfūtei Yūsen, à la fois mécène et participant. Yūsen était un disciple d'Ikeno Taiga, qui était lui-même un ami proche de Minagawa Kien. Un rapide examen du catalogue suffit à nous fournir une longue liste de peintres et de calligraphes remarquables : Minagawa Kien, Poème en écriture cursive sur la pêche en solitaire par une 


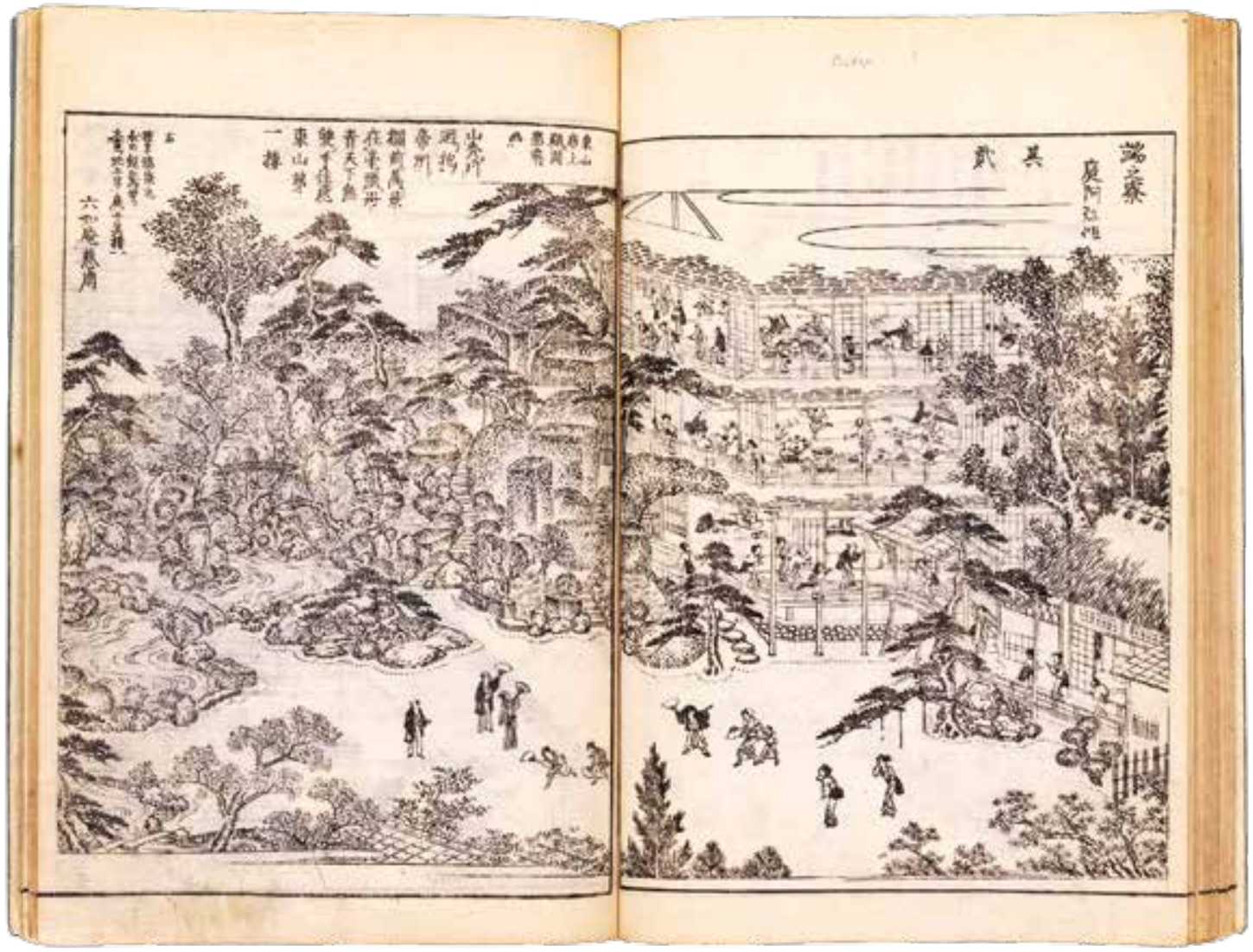

nuit d'été; Itō Jakuchū, Cinq cents Arhat; Go Gekkei (Goshun), Prunier à l'encre; Moine Shōchū (Daiten), Vers en écriture semi-cursive; Maruyama Ōzui, Troupeau

5. "Tan-no-ryō ", dans Oku Bunmei, Akisato Ritō, Nishimura Baikei, Sakuma Sōen, Miyako rinsen meishō zue, 1799. de boufs; Genki, Pivoines; Aoki Shukuya, Paysage à l'encre ; Yamaguchi Soken, Coq et fleurs de prunier ; Kawamura Bunpō, Réunion élégante ; Okamoto Toyohiko, Yang Guifei ; Kimura Kenkadō, Orchidées à l'encre ; Tani Bunchō, Reine mère de l'Ouest; Nagasawa Rosetsu, Su Dongpo ; Matsumoto Guzan, Poème sur un paysage en écriture cléricale; Minagawa Kien, Encres dans l'esprit de Wang Wei. En fin d'ouvrage figurent huit maîtres sous la mention tsuibo no i ( in memoriam »), parmi lesquels " Maruyama Ōkyo, Canards en tons clairs sur soie ", ce qui démontre que les artistes vivants n'étaient pas les seuls représentés. Mort l'année précédente, Ōkyo laissait derrière lui ses " disciples » (Maruyama monjin), nombreux dans le catalogue. Les exposants n'étaient pas tous non plus des adultes ou des hommes : la liste s'ouvre sur un rouleau de Cinq grands personnages en écriture semi-cursive de Miyake Otsuryū, âgé de 7 ans, et il est question plus loin d'une peinture intitulée Chrysanthèmes, en couleurs par une certaine Takeichi, âgée de 10 ans, une autre calligraphie de jeune fille étant encore mentionnée quelques pages plus loin. Le catalogue ne fait pas état de l'âge d'Itō Jakuchū, qui avait 80 ans à l'époque, mais les 95 ans du professeur confucéen Nishiyori Seisai (1702-1797) sont évoqués. Avec un mélange de peintures et de poèmes calligraphiés, l'exposition semble avoir réuni certains des plus grands talents de l'époque ainsi que des intellectuels vénérables, et même des enfants d'un talent exceptionnel. Les thèmes et les styles parcourent toute la gamme de l'art japonais moderne ; si quelques grands noms sont mentionnés d'entrée 

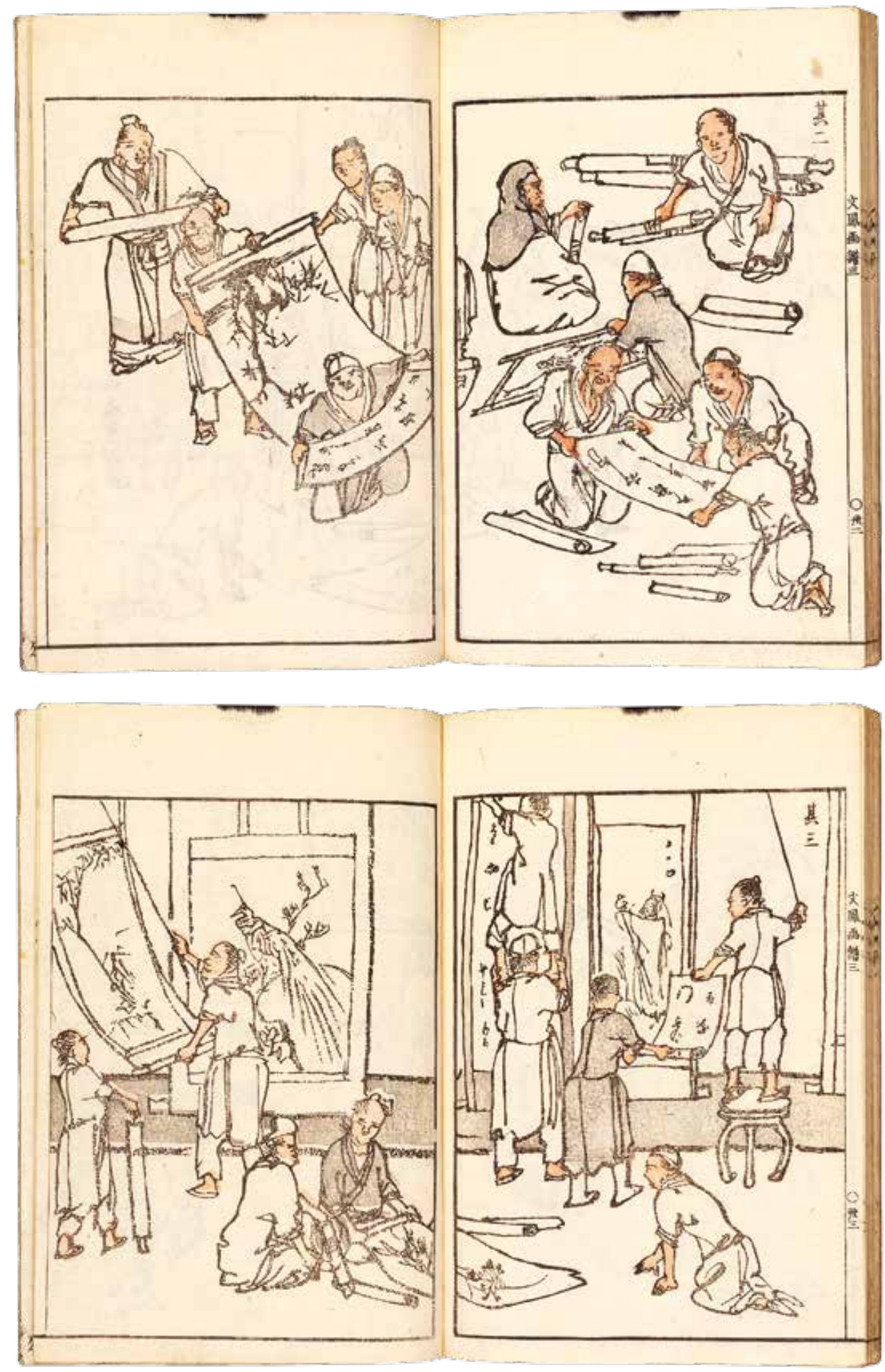
6a-b. Bunpō Kawamura, Shoga tenkan zu [Exposition de calligraphies et de peintures], dans Bunpō gafu [Album de gravures de Bunpō], Kyōto, 1813 de jeu - le peintre de cour Tosa Mitsusada (1738-1806) apparaît ainsi dès les premières pages, aux côtés de celui de Kien -, le catalogue ne donne guère l'impression de suivre un ordre ou une hiérarchie.

Rosetsu apparaît dans deux expositions, mais étant donné ses liens étroits et bien connus avec Kien, il était à n'en pas douter un exposant régulier. Il est mentionné

dans le catalogue de 1796 comme auteur d'un "Su Dongpo, à l'encre ". Cette œuvre aujourd'hui perdue ressemblait peut-être à certaines peintures qu'on lui connaît, représentant un sage chinois en train d'écrire, comme sur le rouleau de la collection Uematsu conservé au musée national de Tōkyō. Ce n'est que deux ans plus tard, en 1798, qu'il a peint les Cinq cents Arhat. Étonnamment, le même thème est inventorié sous le nom de Jakuchū dans le catalogue de 1796, "sur soie ".

En tant qu'adepte du bouddhisme Zen, Jakuchū éprouvait un vif intérêt pour les arhat, et s'il n'est pas généralement considéré comme très doué dans l'art figuratif, il a réalisé une copie intégrale de seize rouleaux attribués au peintre Guanxiu, précédemment mentionné, de la dynastie Tang. Il s'agissait sans doute d'un projet ambitieux pour Jakuchū ; une comparaison avec l'un des originaux conservés témoigne de son effort de fidélité dans la reproduction des robes des arhat, avec leurs plis expressifs, ainsi que des branches rugueuses et irrégulières des arbres (fig. 7). Les arhat de pierre dont Jakuchū a entrepris l'exécution dans les années 1770 étaient alors bien connus : ils furent mentionnés et reproduits dans le guide de Kyōto de 1780, Miyako meisho zue, et firent l'objet d'une célèbre visite de Kien, accompagné d'Ōkyo et de Goshun, au début de l'année 1788. Jakuchū en fit lui-même une série de peintures, dont certaines furent reproduites sous la forme de gravures commercialisées au temple Sekihōji, où ils avaient été installés. Il est difficile de déterminer si la peinture que Jakuchū a présentée en 1796 faisait partie de cette série, mais on peut gager que Rosetsu, ayant participé à la même exposition, a connu les peintures d'arhat réalisées par Jakuchū. Il en a sans doute tiré la matière de sa propre ouvre, où les cinq cents arhat apparaissent désormais réunis dans l'invraisemblable exiguïté d'un carré de trois centimètres sur trois.

C'est dans les années 1790 que Rosetsu et Jakuchū, alors bien plus âgé, ont réalisé certaines de leurs peintures les plus surprenantes sur le plan visuel, rien de tel n'ayant existé auparavant dans la peinture nipponne. D'après l'âge indiqué par Jakuchū à côté de sa signature (82 ans), les paravents intitulés Baleine et éléphant (fig. 8) datent

7. Itō Jakuchū, Arhat, fin du XVIII' siècle, rouleau suspendu, encre sur papier, $113,7 \times 59 \mathrm{~cm}$, Boston, Museum of Fine Arts, William Sturgis Bigelow Collection, inv. 11.6921

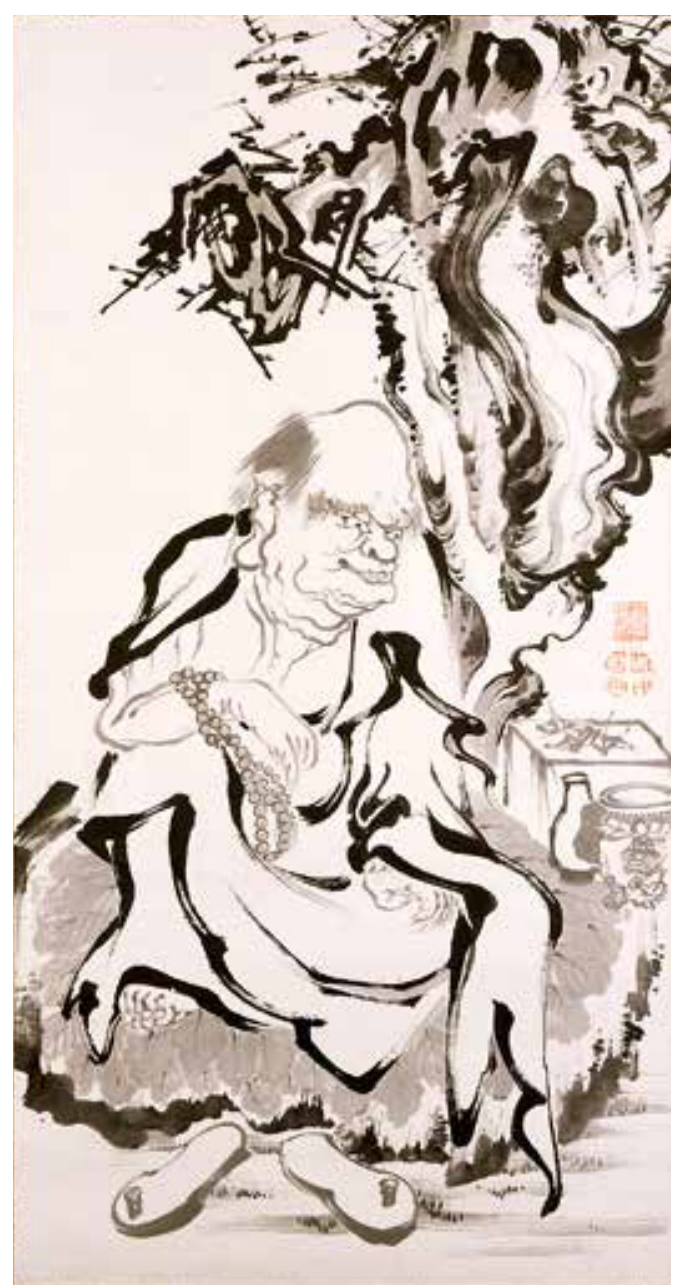


de 1795 (ou de 1797) ${ }^{16}$. Les spécialistes continuent de s'interroger sur la signification de ce couplage, qui représente un parallèle fascinant avec les paravents intitulés Taureau et éléphant de Rosetsu, eux aussi peints à la fin des années 1790. Les deux artistes n'ont-ils été intéressés que par les contrastes formels du noir et du blanc tels qu'ils se manifestent à travers ces animaux démesurés ? Ou faut-il s'orienter vers une lecture bouddhiste alors que la tradition n'établit pas de lien clair, ni avec le Bouddha Sakyamuni (qui monte un taureau), ni avec le Bodhisattva Fugen (qui monte un éléphant) ? Des deux côtés, ces œuvres échappent aux interprétations simplistes.

L'intérêt de ces paravents s'accroît encore si l'on considère que Rosetsu et Jakuchū ne se sont pas limités à des exemplaires uniques : on connaît en effet plusieurs versions de chaque paire. Avant la découverte de Baleine et éléphant en 2008, une paire de paravents quasiment identique était attestée par des photographies dans un catalogue d'enchères qui datait des années 1920, et l'on sait qu'il existe au moins quatre versions des paravents Taureau et éléphant ${ }^{17}$. Plusieurs peintures de Rosetsu existent en plusieurs versions, et si ce phénomène n'a rien d'inédit dans l'histoire de l'art (on pense par exemple à la Vierge aux rochers de Léonard de Vinci), il met en évidence à la fois le degré d'accomplissement technique d'un peintre, le rôle de ses assistants dans l'atelier, et la demande de compositions considérées comme plus ou moins réussies ou désirables ${ }^{18}$. La reproduction de tant de peintures, notamment sur paravents, suggère que le public les connaissait et les désirait au point de commander de nouvelles versions. Les expositions d'Higashiyama ont dû représenter des occasions importantes pour que les clients et les collectionneurs puissent découvrir des artistes qu'ils ne connaissaient pas et des œuvres nouvelles de ceux qu'ils connaissaient déjà.

\section{Les expositions de peintures dans les temples et aux festivals}

Organisées seulement deux fois par an pendant sept ans, les expositions d'Higashiyama n'étaient bien sûr qu'une vitrine parmi d'autres où voir de l'art dans le Kyōto de la fin du XVIII ${ }^{\mathrm{e}}$ siècle. La présentation périodique d'œuvres précieuses conservées dans les temples (bakuryō) représentait depuis longtemps une occasion de voir des peintures anciennes et il semble d'ailleurs qu'elle faisait partie de la formation des peintres ${ }^{19}$. Le Journal du Prince impérial Shinnin de Myōhōin relate ainsi que le $18^{\mathrm{e}}$ jour du $6^{\mathrm{e}}$ mois de Tenmei 7 ( $1^{\mathrm{er}}$ août 1787), " Jakuchū a demandé à ce que quatre de ses disciples puissent voir la présentation faite dans la grande salle Ryūkazō (au temple Myōhōin), ce qui veut dire que nous aurons en tout sept invités avec le groupe d'Ōkyo. La demande lui a été accordée, et j'ai pris mes dispositions pour qu'ils puissent également visiter le jardin ${ }^{20}$. "Si ces événements étaient l'occasion de présenter avant tout des peintures et des calligraphies anciennes, ils inspiraient de toute évidence des œuvres nouvelles, intégrées par les organisateurs dans le parcours. On considère aujourd'hui comme acquis que les rouleaux Tigre et dragon de Rosetsu, aujourd'hui exposés lors des présentations annuelles au temple Daitokuji, ont été inspirés par les rouleaux du peintre chinois Muqi, une source d'influence pour les artistes japonais depuis plusieurs siècles. La retenue et la clarté méticuleuse avec lesquelles Rosetsu a représenté ses créatures attirent notre attention sur la composante météorologique de l'œuvre : le vent qui plisse les vagues, fouette les bambous, chasse les nuages autour du tigre, ainsi que les nuées et les éclairs enveloppant le dragon. L'aisance de Rosetsu dans le lavis sur soie est le principal élément nouveau dans ces peintures, et l'on peut facilement imaginer que les rouleaux anciens de Muqi l'ont poussé à créer une œuvre différente, digne d'un environnement aussi auguste. 

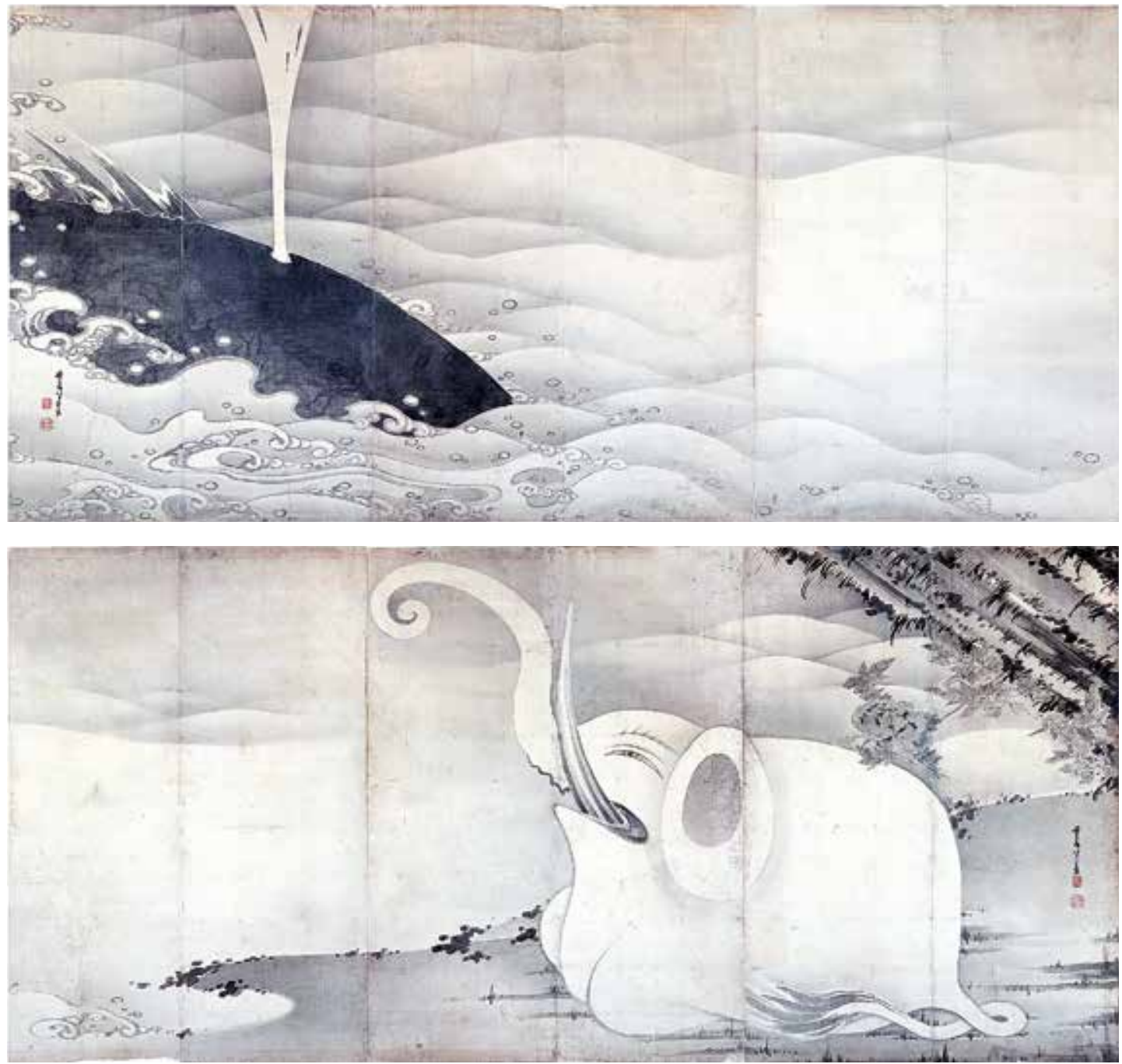

Les festivals, au premier rang desquels celui de Gion, ont dû fournir d'autres occasions importantes de présenter des ouvres audacieuses. La coutume qu'avaient les 8. Itō Jakuchū, Baleine et éléphant, 1797, paire de paravents de six panneaux, encre sur papier, 159,4 × $354 \mathrm{~cm}$ par paravent, Shiga, musée Miho. familles de commerçants d'ouvrir leurs maisons dans ce quartier de Kyōto pour exposer leurs biens et pour démontrer l'importance de la capitale impériale en tant que centre de la production textile de luxe était liée par la pratique courante comme par la tradition orale au goût de l'innovation et de l'originalité visuelles. Si la majeure partie de ce que l'on voit aujourd'hui est banale et de qualité inégale, la présence d'une œuvre comme les Loups au clair de lune de Suzuki Shōnen dans la maison familiale des Ban montre combien les peintres de l'époque cherchaient à satisfaire les aspirations de ces riches clients en matière d'œuvres visuellement saisissantes.

Les documents du milieu du XVIII e siècle indiquent que le " festival des paravents " (byōbu matsuri) ou la "présentation des paravents " (byōbu kazari) étaient bien établis à 


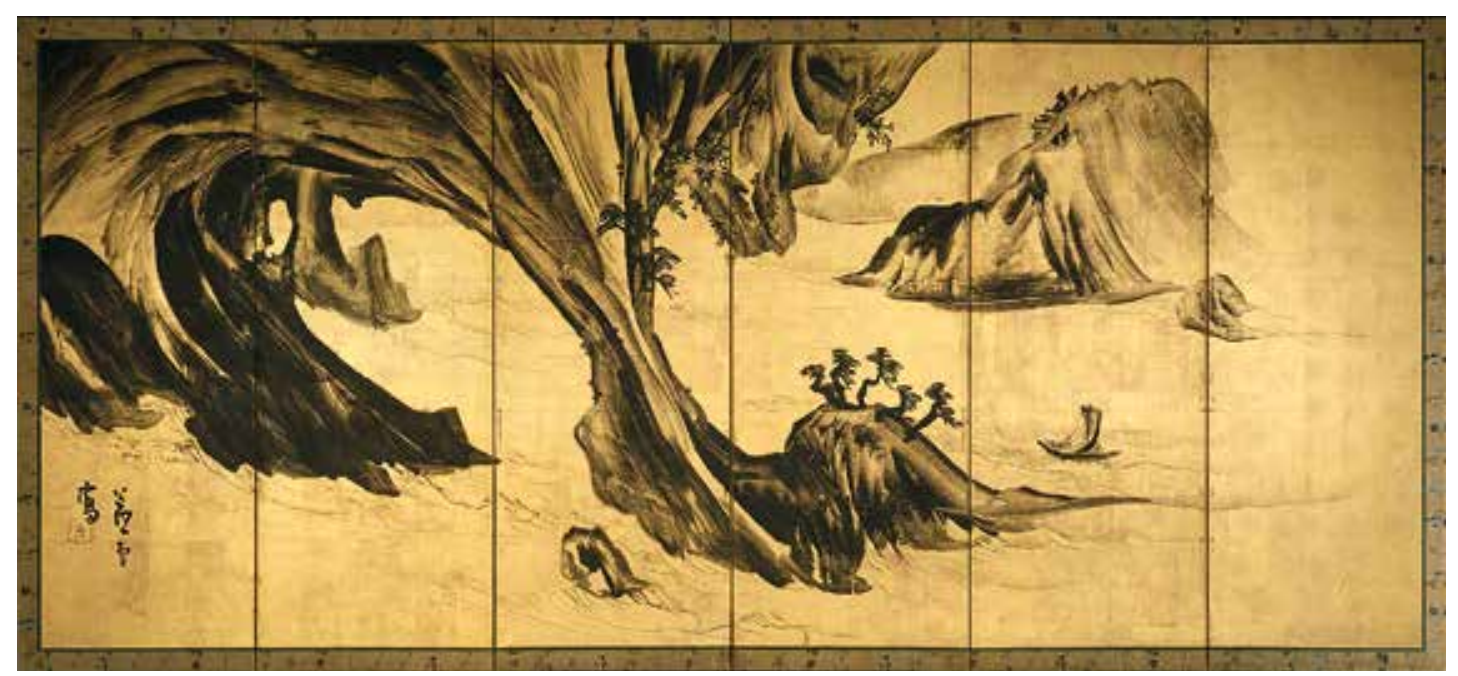

l'époque de Rosetsu. Ainsi peut-on lire dans "Yamaboko yurai ki " [Origine des chars du festival de Gion], un chapitre du livre Gion goryō-e saiki [Compte précis du festival de Gion] paru en 1757 :

Plusieurs jours avant le festival dans les districts où il a lieu, chacun allume d'innombrables lanternes, suspend des étoffes, et expose des paravents d'or et d'argent ainsi que des tapis de laine pour ne pas être en reste par rapport aux ornements des voisins ${ }^{21}$.

Tan Taigi (1709-1771), l'un des tout premiers auteurs de haikai, a laissé un poème révélateur sur les paravents exposés pour le festival du solstice d'été : «Le jour du festival / qui sera juge dans le concours de paravents ? " Le poème de Taigi figurait dans une anthologie de 1777, et j'espère ne pas me tromper en y voyant une association ludique entre le rôle du juge dans un concours de poésie (un uta-awase) et le rôle des spectateurs qui, au festival, offraient leurs commentaires sur les mérites esthétiques des paravents qu'ils avaient sous les yeux ${ }^{22}$. Un texte de la période de Meiji, en 1894, est encore plus clair sur le but de ce genre de décorations festivalières :

Dans les districts où a lieu le festival, des lanternes sont érigées sur tous les avant-toits, décorées de tissus. Les paravents d'or et d'argent sont installés dans les pièces, qui peuvent être décorées de rideaux de perles et de tapis à motif floral. Les lanternes sont allumées et des bouquets de fleurs sont présentés, ainsi que des bonzaïs, pour le divertissement des invités. Leur beauté est tout bonnement saisissante pour les yeux. Le public vient en masse, par dizaines de milliers peut-être, et de loin, pour être témoin du spectacle ${ }^{23}$.

Plusieurs des paravents remarquables de Rosetsu et surtout de son maître Ōkyo proviennent de familles du centre de Kyōto installées dans le commerce de kimonos. Les Pins dans la neige d'Ōkyo, qui font partie de la collection Mitsui, en donnent un exemple majeur, et les Nishimura, propriétaires des ateliers Chisō, ont encore en leur possession des paravents parmi lesquels certains sont de Rosetsu et d'Ōkyo. De telles familles avaient des entrepôts entièrement remplis d'œuvres d'art, et le caractère novateur d'une œuvre augmentait sans doute son attrait lorsqu'elle était d'un peintre réputé.

Parmi les dernières œuvres de Rosetsu, Paysages avec des personnages chinois (fig. 9a-b), ultime recherche dans le genre de la peinture de paysage, apparaît comme l'une des plus étonnantes. Ces paravents dorés à la feuille d'or et peints à l'encre monochrome 


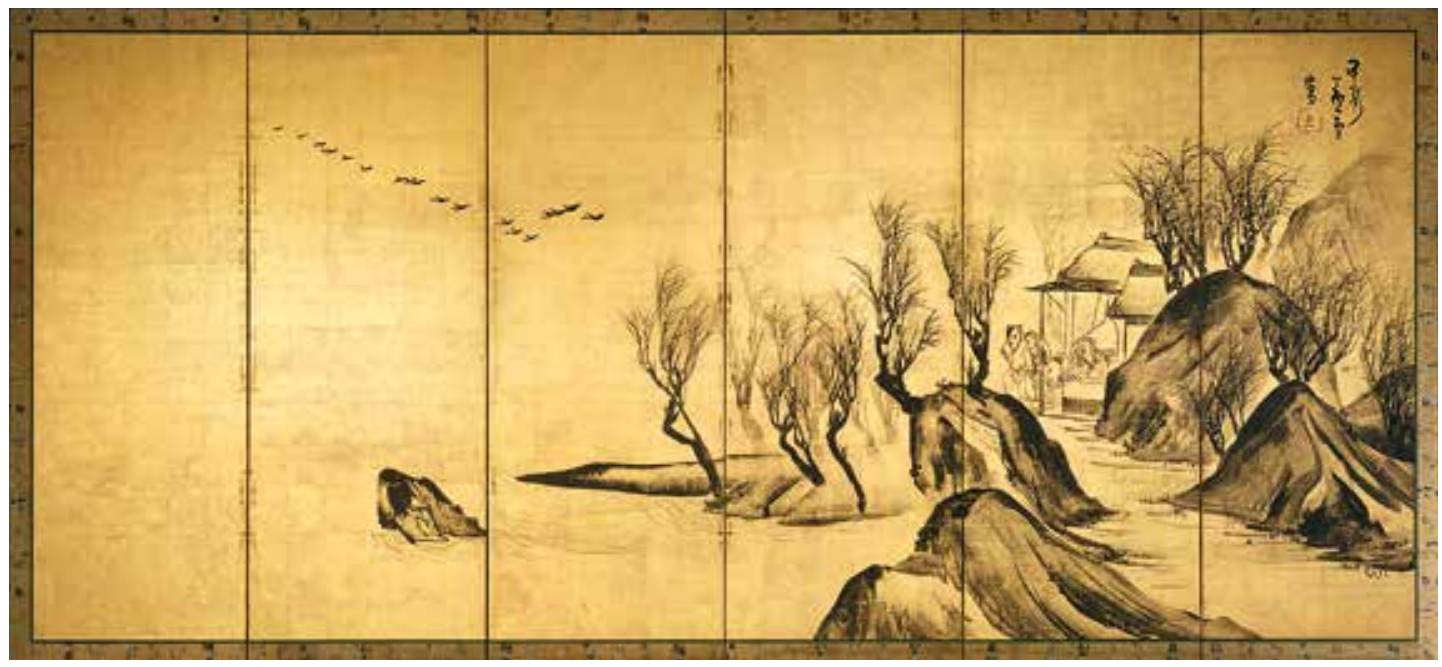

amplifient l'abstraction de ses paysages antérieurs. Ils puisent dans une imagerie familière issue de la littérature chinoise classique, avec le vol d'oies et les saules de Tao Qian sur le paravent de droite et, sur celui de gauche, les abrupts précipices qui évoquent la

9a-b. Nagasawa Rosetsu, Paysage avec personnages chinois, vers 1789-1799, paire de paravents de six panneaux, encre sur papier doré, 154,6 × 356 cm par paravent, New York, Metropolitan Museum of Art.

Falaise rouge de Su Shi. La spontanéité des paysages, les mouvements amples du large pinceau sur la feuille d'or, et les nombreux endroits où l'encre a coulé, suggèrent que Rosetsu les a peints devant une assistance ${ }^{24}$. Si ces peintures évoquent son séjour sur certaines des côtes pittoresques du Japon, elles témoignent aussi de son extraordinaire confiance en lui, dont les récits sur la dernière période de sa vie font souvent état.

Des paravents comme Baleine et éléphant de Jakuchū, Taureau et éléphant de Rosetsu, ou encore Paysages avec des personnages chinois, donnent ainsi corps à l'allusion de Minagawa Kien à des œuvres " originales et bizarres " dans une décennie où les peintres rivalisaient d'ingéniosité et d'inventivité pour réaliser des compositions toujours plus saisissantes, les panneaux articulés du paravent ayant le format idéal pour toucher de larges publics. Les peintres de Kyōto au XVIII ${ }^{e}$ siècle s'essayaient déjà depuis des décennies à des représentations surprenantes, comme le montrent certaines des œuvres de Soga Shōhaku et d'Ikeno Taiga, entre autres. L'incendie de Tenmei 8 (1788) a sans aucun doute entraîné une recrudescence des commandes dans les années qui ont suivi, de la part des habitants de Kyōto mais surtout des commerçants et des artisans du centre de la capitale, qui étaient aussi le moteur de son économie. Le renouveau du mécénat a de toute évidence représenté une chance pour l'originalité : si l'atelier d'Ōkyo a occupé une position dominante jusque dans les années 1790, des images de peintres indépendants quoique redevables envers le maître, comme Rosetsu et Ganku, démontrent qu'il y avait aussi une demande pour quelque chose de différent et de plus novateur. C'est justement à cette époque, en 1796 et en 1797, que le vénérable Itō Jakuchū a peint ses paravents Baleine et éléphant et Légumes, et les productions d'atelier comme les paravents " mosaïques " qui lui sont associés datent aussi, certainement, de la même période. Les peintres furent soutenus dans la diffusion et la promotion de leurs œuvres par les expositions semestrielles d'Higashiyama, ainsi que par l'industrie croissante de manuels de peinture imprimés (gafu). L'invention et l'originalité étaient les nouveaux critères esthétiques. 


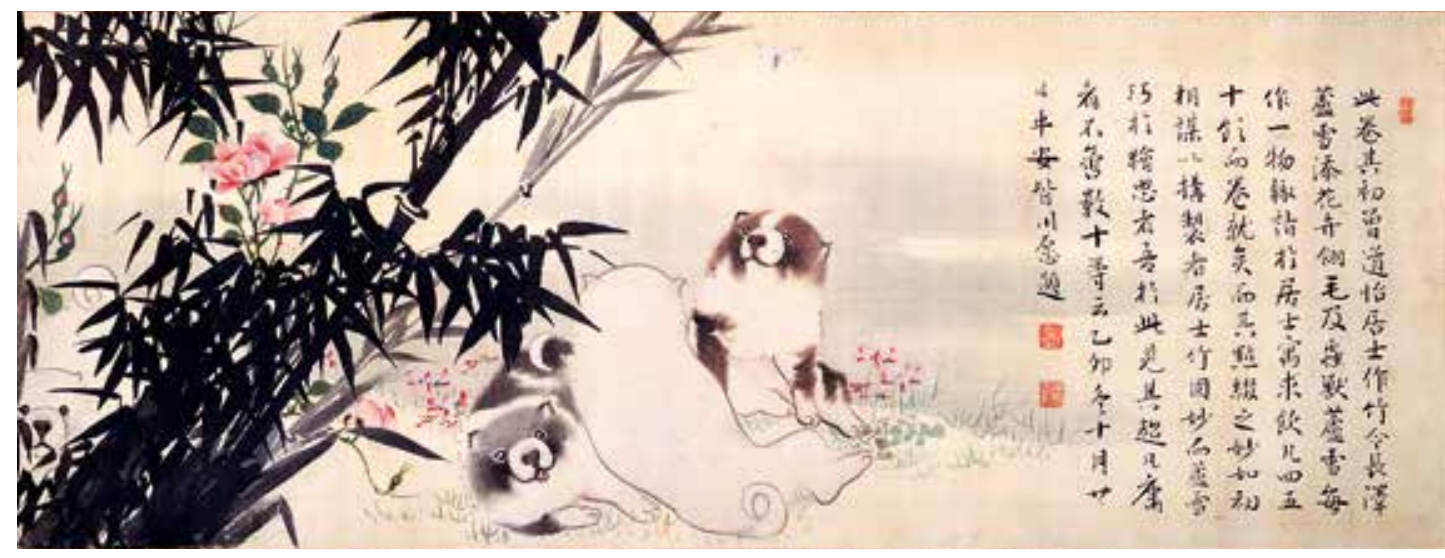

10. So Dōi et Nagasawa Rosetsu, Fleurs, oiseaux, et animaux, 1795, rouleau, encore et couleurs sur soie, $33 \times 371,4 \mathrm{~cm}$, musée des Beaux-Arts de Chiba. Préface de Minagawa Kien.
Mes recherches m'ont montré qu'une grande partie de l'originalité des œuvres de Rosetsu tient à la manière dont il les a exécutées. Les inscriptions, colophons et écrits complets de Kien ont constitué une source inestimable, car ses commentaires portent souvent sur le processus pictural lui-même ${ }^{25}$. Ainsi Kien précise-t-il dans la préface d'un rouleau que Rosetsu, pour ajouter

des fleurs, des oiseaux et des animaux colorés à des bambous préalablement peints à l'encre par quelqu'un d'autre, a exigé plus d'une rasade de saké et plus d'une séance avant de finir le travail (fig. 10). Dans un poème sur un paravent de 1796 aujourd'hui perdu, Kien s'émerveille de la manière dont Rosetsu a réussi à recouvrir la surface des panneaux par la peinture d'une puce monstrueusement magnifiée (un éventail peint nous donne une idée de ce à quoi elle ressemblait). Si Kien ne touche pas un mot de Vol de grues au-dessus du mont Fuji (fig. 11), cette œuvre semble nous inviter à la regarder de près et à nous demander comment Rosetsu a pu figurer ces grues, avec leurs corps de soie vierge de toute peinture devant les pentes grises, escarpées, du mont Fuji.

Les Cinq cents Arhat offrent un parfait exemple de la manière dont Rosetsu attire notre attention sur le processus pictural lui-même. Nul doute qu'il ne s'associe à la prouesse surhumaine des arhat à travers cet effort singulier d'en représenter cinq cents dans un carré de trois centimètres sur trois, sans aucun précédent dans la tradition iconographique en la matière. On retrouve trace de cette prouesse dans certains jugements posthumes, parmi lesquels ce témoignage d'Anzai Un'en qui, au XIXe siècle, a raconté que " Rosetsu, ayant entendu un homme se déclarer incapable de décrire ses œuvres parce qu'il n'y avait rien vu de fin, peignit cent oiseaux dans un carré de trois centimètres sur trois ; par quoi cet homme admit qu'il n'avait vraiment rien à envier à Ōkyo ${ }^{26}$ ". Comme il avait passé la quarantaine d'années et qu'il semblait souffrir d'une insuffisance à un oil, Rosetsu a probablement réalisé les arhat à l'aide d'une loupe ou d'un microscope, un outil dont on sait par Minagawa Kien qu'il s'est servi pour d'autres œuvres. L'intervention d'un tel outil a sans doute augmenté l'attrait de l'œuvre, étant donné les associations que le spectateur informé pouvait faire avec des sommités comme Kimura Kenkadō, possesseur d'une loupe.

Le sixième mois de cette année, 1799, Rosetsu est mort au cours d'un voyage à Ōsaka. Comme, dans cette petite peinture, l'artiste avait donné tout son cœur et toute son âme, elle a été donnée au temple où se sont déroulées ses funérailles, à Ōsaka, pour qu'elle y soit conservée à perpétuité comme l'un de ses objets sacrés. Une version 
préparatoire de cette peinture, plus ancienne, a cependant refait surface. Elle a été achetée par le commerçant An [Yasu] Kisei, qui a décidé de la faire monter dans un rouleau de la taille adéquate. M'ayant présenté ce rouleau, il m’a demandé de mettre par écrit les renseignements sur cette œuvre et sur son acquisition. J'ai beau ne pas encore être ce que l'on appelle un vieux sage, je semble recevoir de nombreuses demandes de ce genre - sans doute est-ce là un châtiment divin. Rosetsu aimait les grands formats et nombre de ses œuvres sont passablement gigantesques, en tout cas il n'y en a pas d'autre aussi minuscule que celle-ci. C'est la raison pour laquelle cette peinture, aujourd'hui dans la collection de curiosités de Yasu Kisei, a dix fois plus de prix que les autres.

Automne, $11^{\mathrm{e}}$ jour, $8^{\mathrm{e}}$ mois de l'ère Kansei $(1799)^{27}$

La brève allusion de Kien à la mort prématurée de Rosetsu à Ōsaka en 1799 est confirmée par le registre des décès du temple Ekōin, qui précise qu'il est mort le huitième jour du sixième mois de l'année ${ }^{28}$. L'existence d'une deuxième stèle funéraire au temple Tenryūin à Ōsaka vient encore étayer le récit de Kien, même si les détails concernant la mort de Rosetsu demeurent obscurs ${ }^{29}$. Nous ne pouvons qu'imaginer les merveilles que Rosetsu aurait réalisées s'il avait vécu plus longtemps.

Les expositions temporaires sont assez rapidement devenues des événements périodiques à Kyōto, puis à Edo et dans d'autres villes, bien avant que ce phénomène ne prenne une forme institutionnelle à la fin du XIX ${ }^{e}$ siècle avec l'avènement des musées et de l'époque moderne. De tels dispositifs de présentation publique allaient continuer à influencer les esprits en matière d'innovation artistique, en particulier picturale. Parallèlement, les expositions commémoratives et les manuels imprimés ont renforcé les rapports d'affiliation au sein de lignées de peintres - autrement dit la tradition - sous la dynamique combinée de la participation collégiale et de la diffusion par l'impression des styles des vieux maîtres comme Maruyama Ōkyo ou Sakai Hōitsu. La riche décennie de l'ère Kansei représente un pic de créativité dont les Cinq cents Arhat de Rosetsu sont, dans une certaine mesure, emblématiques, en lien avec cette pratique nouvelle qu'était l'exposition de peintures originales. La question de l'innovation dans la peinture du XIX siècle devra faire le sujet d'une étude à part, mais ce qui d'ores et déjà me frappe, c'est que l'innovation, chez les artistes du XVIII ${ }^{\mathrm{e}}$ siècle comme Ōkyo, Rosetsu et Shōhaku, est devenue elle-même la source d'une tradition à perpétuer sans grand changement pour les artistes ultérieurs, comme Ōshin et Roshū, ou, dans le cas de Shōhaku, Yokoyama Kazan. Une étude approfondie permettra de démontrer que

11. Nagasawa Rosetsu, Vol de grues au-dessus du mont Fuji, 1794, rouleau suspendu, encre et ton clair sur soie, $157 \times 70,5 \mathrm{~cm}$, collection privée.

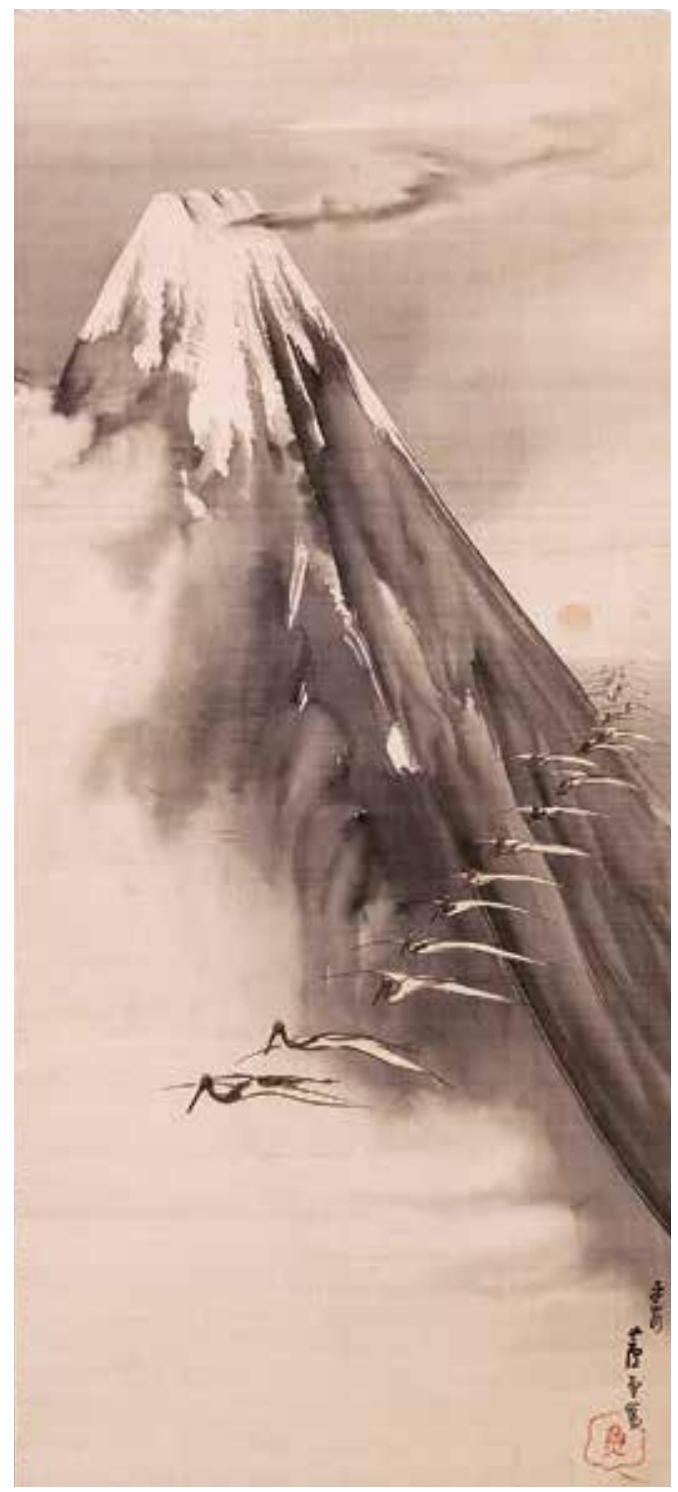


l'éclectisme, les échanges de styles, et la perpétuation de lignées anciennes comme celle de Maruyama-Shijō dans les décennies qui ont mené à la Restauration de Meiji ont dérivé de l'institutionnalisation même des modes d'exposition qui se sont avérés fertiles pour l'imagination de Rosetsu et de ses contemporains.

Cette contribution a été traduite de l'anglais par Étienne Gomez. 


\section{Matthew McKelway}

Matthew McKelway est professeur d'histoire de l'art japonais à Columbia University (chaire Takeo and Itsuko Atsumi). Il a écrit sur les paravents peints à Kyōto (rakuchū rakugai zu) et le développement de la peinture de genre à l'époque moderne au Japon, sur la peinture des écoles Kanō, Rinpa, et les peintres " individualistes " de Kyōto au XVIII" siècle. Il a été professeur invité à la Freie Universität Berlin, à l'Universität Heidelberg, à la Seijō University, et à l'université Paris 1 - Panthéon-Sorbonne.

\section{NOTES}

1. Minagawa Kien et Takahashi Hiromi, Kien shibunshū, Tōkyō, Perikansha, 1986, p. 293-294.

2. Rosetsu-D'un pinceau impétueux, Zurich, musée Rietberg, 5 septembre -4 novembre 2018. Voir Matthew McKelway et Khanh Trinh (dir.), Rosetsu: Ferocious Brush, cat. exp. (Zurich, Museum Rietberg, 2018), Munich / Londres / New York / Zurich, Prestel / Museum Rietberg, 2018.

3. The Lineage of Eccentrics: The Miraculous World of Edo Painting, Tōkyō, Tōkyō-to Bijutsukan [musée d'art métropolotain de Tōkyō], 9 février - 7 avril 2019.

4. Voir Tsuji Nobuo et Murakami Takashi, Nobuo Tsuji vs. Takashi Murakami: Battle Royale! Japanese Art History, Christopher Stephens et Yuko Sakata (trad. angl.), Tōkyō, Kaikai Kiki, Ltd., 2017, p. 216-217, 225.

5. Parmi les travaux les plus récents, I'article que Sugimoto Yoshihisa a consacré à Maruyama Ōkyo et aux projets iconographiques de son atelier pour le prince-abbé Shinnin de Myōhōin montre l'intérêt potentiel de cet angle d'étude. Voir Sugimoto Yoshihisa, "Myōhōin monzeki Shinnin Hosshinnō to Maruyama Ōkyo no monjin tachi: Maruyama Ōzui, Goshun, Nakamura Sokubyō, Nagasawa Rosetsu, Genki »[Prince Abbot Shinnin et les suiveurs de Maruyama Ōkyo : Maruyama Ōzui, Goshun, Nakamura Sokubyō, Nagasawa Rosetsu, Genki], dans Kurokawa Kobunka Kenkyūjo kiyō [Research Journal of the Kurokawa Institute of Ancient Cultures], n 16, 2017, p. 99-170.

6. François Lachaud, Le vieil homme qui vendait du thé : excentricité et retrait du monde dans le Japon du XVIII siècle, Paris, Cerf, 2010.

7. Tsuji Nobuo, Lineage of Eccentrics: Matabei to Kuniyoshi, Aaron M. Rio (trad. angl.), Tōkyō, Kaikai Kiki, Ltd., 2012, p. 6.

8. Ces commentaires d'ordre général sur les arhat et leurs représentations en Asie de l'Est sont tirés de Stephen Little, "The Arhats in China and Tibet ", dans Artibus Asiae, vol. 52, no 3/4, 1992, p. 255-281.

9. Pour une étude précise au sujet des représentations d'arhat chinois, voir Evelyne Mesnil, "Les Seize "Arhat" dans la peinture chinoise ( $\left(\mathrm{VII}^{\mathrm{e}}-\mathrm{X}^{\mathrm{e}} \mathrm{s}\right.$.) et les collections japonaises : prémices iconographiques et stylistiques", dans Arts Asiatiques, vol. 54, 1999, p. 66-84.
10. Selon Huang Xiufu ( $\mathrm{X}^{\mathrm{e}} \mathrm{-Xl^{ \textrm {e } }}$ siècles), auteur de Yizhou Ming hua lu (益州名画録), Guanxiu a représenté ses arhat « avec leurs sourcils broussailleux et leurs grands yeux, leurs mâchoires tombantes et leurs nez saillants, appuyés contre des pins et des rochers ou assis dans un cadre montagneux, rendant à la perfection la particularité de leurs traits tartares ou indiens. Quand je l'ai interrogé à leur sujet, il m’a répondu: "Je les ai vus en rêve." "Voir Susan Bush et Hsio-yen Shih, Early Chinese Texts on Painting, Cambridge, Harvard University Press, 1985, p. 105.

11. La traduction de ce passage est basée en partie sur celle qui figure dans Tsuji et Murakami, 2017, cité n. 4, p. 219. La version originale en langue littéraire chinoise figure dans Minagawa et Takahashi, 1986, cité n. 1, p. 293-294.

12. Voir Robert Campbell [Robaato Kyanberu], « Kanshō no nagare: Shogakai yonseki sono ichi - Ginkakuji Higashiyamadono sanbyaku kaiki » [La contemplation vagabonde : quatre expositions de calligraphie et de peinture, $\mathrm{n}^{\circ} 1$ - en mémoire du $300^{\mathrm{e}}$ anniversaire d'Ashikaga Yoshimasa (Higashiyamadono) à Ginkakujil, dans Bungaku [Littérature], vol. 8, n² 2, printemps 1997, p. $140-141$

13. Tanabe Nahoko, "Aimi bunko zō Shinshoga tenkan mokuroku honkoku to kaidai: Kansei-ki no Kyoto shogadan to Minagawa Kien (jō) » [Réimpression et synopsis des catalogues des nouvelles expositions de peinture et de calligraphie dans les archives d'Aimi : Minagawa Kien et les cercles de peinture et de calligraphie à Kyōto à l'ère Kansei], dans Bunken kenkyū [Études des documents historiques], $n^{\circ}$ 41, 2003.

14. Tanabe, 2003, cité n. 13, p. 58-60.

15. Voir Tim Clark, "The Jakuchū Memorial Exhibition of 1885 ", dans Yukio Lippit (dir.), The Artist in Edo, actes du colloque (Washington D.C, Center for Advanced Study in the Visual Arts, National Gallery of Art / Freer Gallery of Art and Arthur M. Sackler Gallery, Smithsonian Institution, 2012), Washington DC / New Haven, National Gallery of Art (coll. "Studies in the History of Art ", 80) / Yale University Press, 2018, p. 254-255.

16. Diverses hypothèses ont été avancées pour expliquer les âges indiqués par Jakuchū sur ses dernières œuvres, en particulier du fait que sur certaines signatures il prétend avoir quatre-vingt-six ans ou davantage, alors qu'il n'avait pas encore atteint cet âge à sa mort en 1800. Selon Kano Hiroyuki, passé soixante ans, Jakuchū a ajouté un an à chaque nouveau nom de règne (nengō). Selon Tsuji Nobuo, cependant, Jakuchū n'aimait tout simplement pas le chiffre quatre et s'attribuait des âges différents, notamment soixante-quinze ans, afin de l'éviter. Tsuji, Kisō no keifu ten, cat. exp. (Tōkyō, Tōkyō-to Bijutsukan [musée d'art métropolitain de Tōkyō]), Tōkyō, Shōgakkan, 2019, p. 260.

17. Voir Matthew McKelway, "After Rosetsu », dans Impressions, vol. 41, 2020, p. 110-111.

18. La formule "motome ni ōjite " ("répond à une demande ») figure sur un si grand nombre d'œuvres reproduites par différents artistes qu'elle témoigne sans doute de l'existence de versions alternatives. 
19. 曝涼. Pour une analyse détaillée de ces pratiques au monastère Daitokuji, voir Gregory P. A. Levine, Daitokuji: the Visual Cultures of a Zen Monastery, Seattle, University of Washington Press, 2006.

20. D'après Shinnin hosshinnō onjiki nikki [Diary of Imperial Prince Shinnin of Myōhōin], cité dans Jens Bartel, "Style, Space and Meaning in the Large-Scale Landscape Paintings of Maruyama Ōkyo (1733-1795) ", Ph.D. dissertation, Columbia University, 2019, Appendix B, 365 .

21. Voir "Yamaboko yurai ki », p. 4 (recto), dans Gion goryō-e saiki [Compte précis du festival de Gion], Kyōto, Yamamoto Chōbei, Hōreki 7 (1757) ; voir aussi Kano Hiroyuki, "Byōbu matsuri: Gion-e no haikei » [Le festival de paravents : contexte du festival de Gion], dans Akai Tatsurō et Nakajima Junji (dir.), Kinsei fūzoku zufu 8: Sairei (1) [Recueil des premières peintures de genre modernes 8 : Festivals (1)], Tōkyō, Shōgakkan, 1982, p. 118.

22. Le poème de Taigi a d'abord été versé au dossier par Takeda Tsuneo, cité ici par Kano, 1982, cité n. 21.

23. Kyōto Gion-e zue, cité par Kano, 1982, cité n. 21, p. 117.

24. J'ai défendu cette hypothèse dans un article consacré au Paysages avec des personnages chinois. Voir Matthew P. McKelway, "Rosetsu's Red Cliffs: Medium and Meaning in Late Edo-Period Painting ", dans Lippit, 2018, cité n. 15, p. 15-46.

25. Ibidem.

26. Kinsei meika shoga dan, cité intégralement dans McKelway et Trinh, 2018, cité n. 2.

27. Ibidem, p. 219. Pour le texte original, se reporter à Minagawa et Takahashi, 1986, cité n. 1, p. 293-294.

28. Yamakawa Takeshi, " Nagasawa Rosetsu denreki » [Biographie de Nagasawa Rosetsu], dans Kokka, vol. 860, novembre 1963, p. 60.

29. Nagasawa Roshū a érigé la stèle funéraire aujourd'hui à Tenryūin en 1838 en remplacement d'une ancienne pierre tombale au temple voisin de Jikishi'an, sans doute lié à Shikyō Eryō (1722-1787), moine du sous-temple Kaifukuin du monastère zen Myōshinji, pour qui Rosetsu a peint une image murale d'un dragon à Jikishi'an, son ermitage, en 1783. Elle est restée à Jikishi'an jusque peu avant 1901, d'où l'on sait qu'elle a été déplacée à Tenryūin en raison de la fermeture du temple. Voir Okada Hideyuki, "Nagasawa Rosetsu no denki to sakuhin: shin shiryō o kuwaete» [Le vie et l'œuvre de Nagasawa Rosetsu : à la lumière d'œuvres récemment découvertes], dans Miho Museum, Nagasawa Rosetsu: ki wa shin nari / Nagasawa Rosetsu: The Fanciful Painter, cat. exp. (Kōka, musée Miho, 2011), Kōka, musée Miho, 2011, p. 266-267. 